\title{
Correlation between liquefaction resistance and shear wave velocity of granular soils: a micromechanical perspective
}

\author{
X. M. XU* X $^{*}$, D. S. LING*, Y. P. CHENG $\dagger$ and Y. M. CHEN*
}

\begin{abstract}
The shear wave velocity method has become an increasingly popular means to evaluate the liquefaction potential of granular soils. Understanding the fundamental mechanism underlying existing empirical or semi-empirical relationships is important for better assessing their reliability. This paper presents a particle-scale study of the correlation between cyclic resistance ratio (CRR) and the shear wave velocity corrected for overburden stress $\left(V_{\mathrm{s} 1}\right)$. The discrete-element method was used to simulate a series of undrained stress-controlled cyclic triaxial tests together with shear wave velocity $\left(V_{\mathrm{s}}\right)$ measurements. Discrete-element method modelling with various relative densities, confining pressures and micro-parameters was performed under various cyclic stress ratios (CSRs), and the onset of liquefaction was illustrated through both macroscopic and microscopic responses, for example, inferred excess pore-water pressure, mechanical coordination number and redundancy index. The inter-particle friction was identified as the key micro-parameter that governs the liquefaction resistance of granular soils. A micro-scale $\mathrm{CRR}-V_{\mathrm{s} 1}$ correlation considering two independent micro-parameters, inter-particle friction and particle shear modulus, was then obtained and further validated with the outcomes from three dynamic centrifuge model tests performed on silica sand no. 8. This study demonstrates that the CRR $-V_{\mathrm{s} 1}$ correlation is particle specific, thus soil specific, and the particle mechanical properties should be included in the $V_{\mathrm{s}}$-based method for future liquefaction evaluation of granular soils.
\end{abstract}

KEYWORDS: centrifuge modelling; discrete-element modelling; particle-scale behaviour; liquefaction

\section{INTRODUCTION}

The simplified method pioneered by Seed \& Idriss (1971), based on standard penetration test (SPT) data, has become the standard practice for evaluating the liquefaction potential of granular soils (Youd \& Idriss, 2001; Youd et al., 2001). Earthquake-related soil liquefaction is discussed in relation to the cyclic stress ratio (CSR) induced by ground shaking at some depth in the ground

$$
\mathrm{CSR}=\frac{\tau_{\mathrm{av}}}{\sigma_{\mathrm{v} 0}^{\prime}}=0.65\left(\frac{a_{\mathrm{max}}}{\boldsymbol{g}}\right)\left(\frac{\sigma_{\mathrm{v} 0}}{\sigma_{\mathrm{v} 0}^{\prime}}\right) r_{\mathrm{d}}
$$

where $\tau_{\mathrm{av}}$ is the average equivalent uniform cyclic shear stress caused by the earthquake and is assumed to be 0.65 of the maximum induced stress; $\boldsymbol{g}$ is the acceleration of gravity; $\sigma_{\mathrm{v} 0}$ and $\sigma_{\mathrm{v} 0}^{\prime}$ are total and effective vertical overburden pressures, respectively; and $r_{\mathrm{d}}$ is stress reduction coefficient to adjust for the flexibility of the soil profile.

The key issue is to characterise the capacity of soil to resist liquefaction based on various routinely used field or laboratory techniques (e.g. SPT, cone penetration test (CPT), Becker hammer test (BHT) and shear wave velocity $\left(V_{\mathrm{s}}\right)$ measurement). Among them, the shear wave velocity corrected for overburden stress $\left(V_{\mathrm{s} 1}\right)$ is considered to offer

Manuscript received 4 April 2014; revised manuscript accepted 13 January 2015. Published online ahead of print 26 March 2015.

Discussion on this paper closes on 1 October 2015, for further details see p. ii.

* Key Laboratory of Soft Soils and Geoenvironmental Engineering of Ministry of Education, Department of Civil Engineering, Zhejiang University, Hangzhou, China.

$\dagger$ Department of Civil, Environmental and Geomatic Engineering, University College London, London, UK.

t Department of Engineering, University of Cambridge, Cambridge, UK. geotechnical engineers a promising alternative to evaluate the liquefaction resistance of granular soils. This is especially true for sites underlain by soils that are difficult to penetrate or sample (Andrus \& Stokoe, 2000; Kayen et al., 2013). The $V_{\mathrm{s}}$-based simplified method has attracted an increasing number of studies, and various 'boundary curves' have been relatively well established on the basis of either field data (Robertson et al., 1992; Andrus \& Stokoe, 2000; Juang et al., 2001; Andrus et al., 2004; Juang et al., 2005; Kayen et al., 2013), or laboratory studies (Dobry et al., 1981; de Alba et al., 1984; Tokimatsu \& Uchida, 1990; Chen et al., 2005; Wang et al., 2006; Zhou \& Chen, 2007; Baxter et al., 2008; Zhou et al., 2010; Ahmadi \& Paydar, 2014).

Note that these $V_{\mathrm{s}}$-based correlations were developed from empirical or semi-empirical evaluation of field observations and laboratory test data following the general format of the simplified procedure by Seed \& Idriss (1971). Their fundamental mechanisms are still open to question. First, the shear wave velocity measurements are made at small strains, whereas pore-water pressure build-up and liquefaction are medium-to-high-strain phenomena (Roy et al., 1996). Whether there is a natural link between these two different characterising variables remains unknown. Second, the uniqueness of this correlation for all types of granular soils is also questionable. Although both $V_{\mathrm{s}}$ and liquefaction resistance were reported to be similarly influenced by many of the same macroscopic factors (Andrus \& Stokoe, 2000), their parametric laws may be quite different. Taking the relative density for an example, previous studies showed that it has a very strong effect on the liquefaction resistance (Seed \& Idriss, 1971), while it has a weak effect on $V_{\mathrm{s}}$. This implies that liquefaction resistance may not uniquely correlate with $V_{\mathrm{s}}$ for multiple soils (Baxter et al., 2008), as indicated by the weakness of the $V_{\mathrm{s}}$-based correlation reported by Liu \& Mitchell (2006). More recently, Kayen et 
al. (2013) reported that around 50 non-liquefied sites out of a global catalogue of 422 case histories were misclassified, with their data mainly located in the lower intensity zone (i.e. cyclic stress ratio, CSR $\leqslant 0 \cdot 20$ ). One possible explanation for these observations is that $V_{\mathrm{s}}$-based correlations are soil specific; however, establishing individual relations would be rather costly and time consuming (Tokimatsu \& Uchida, 1990; Zhou \& Chen, 2007; Baxter et al., 2008; Zhou et al., 2010; Ahmadi \& Paydar, 2014).

The shear wave velocity is well known as a comprehensive representative metric for characterising the current state of granular soils, which is highly dependent on both macroscopic parameters (e.g. stress state, void ratio or relative density) and microscopic parameters (e.g. particle properties, fabric and coordination number) (Hardin \& Richart, 1963; Tatsuoka, 1999; Yimsiri \& Soga, 2000; Agnolin \& Roux, 2007; Clayton, 2011). The liquefaction resistance in a soil deposit, commonly quantified by the cyclic resistance ratio (CRR), also depends on its current state (Seed \& Idriss, 1971; Tokimatsu \& Uchida, 1990; Chen et al., 2005; Wang et al., 2006; Baxter et al., 2008; Ahmadi \& Paydar, 2014). CRR is generally estimated by performing cyclic triaxial tests on reconstituted samples following a given stress path (e.g. a series of sinusoidal cyclic stress cycles), and is defined as the CSR to cause initial liquefaction after a certain number of cycles (e.g. 15 cycles corresponding to an earthquake magnitude, $M_{\mathrm{w}}=7 \cdot 5$ ). Exploring the underlying fundamental mechanisms of CRR and $V_{\mathrm{s}}$ at both macro and micro level is essential to establish their correlation for the purposes of liquefaction evaluation of granular soils. However, it is still a great practical challenge for experimenters to examine and accurately quantify the intricate characteristics of internal soil structure at the micro scale.

Numerical simulations using the distinct-element method (DEM) pioneered by Cundall \& Strack (1979) can offer some micromechanical insights to understand the mechanical properties of granular soils (Thornton, 2000; Cheng et al., 2004; Soga \& O'Sullivan, 2010; O'Sullivan, 2011; Zhao \& Guo, 2013). Its applicability to the modelling of the cyclically induced liquefaction behaviour of granular soils shearing at constant volume has been demonstrated (Shafipour \& Soroush, 2008). Ng \& Dobry (1994) were among the first researchers who studied the responses of both two-dimesional (2D) disc and three-dimensional (3D) sphere assemblies in undrained cyclic simple shear loading conditions. Their results qualitatively agreed with physical tests on sand. Recently, although there have been a number of both 2D (Sitharam, 2003) and 3D (O'Sullivan et al., 2008; Sitharam et al., 2009; Soroush \& Ferdowsi, 2011) numerical simulations that have investigated liquefaction phenomenon based on strain-controlled loading, the authors are not aware of any DEM studies that have explored the micromechanics both of liquefaction and of CRR in a stress-controlled manner, and further developed a CRR $-V_{\mathrm{s} 1}$ correlation by microscopically measuring the $V_{\mathrm{s}}$ of granular soils.

Following similar procedures to laboratory tests, this paper presents numerical simulations using a particle-scale DEM investigation of the $\mathrm{CRR}-V_{\mathrm{s} 1}$ correlation, by conducting a series of undrained stress-controlled cyclic triaxial test simulations, together with $V_{\mathrm{s}}$ measurement. DEM specimens with various initial relative density, confining pressure and microparameters are tested under various cyclic stress amplitudes. The onset of liquefaction is illustrated through both macroscopic and microscopic responses. The key micro-parameters that govern the magnitude of CRR are identified, and a micro-scale $\mathrm{CRR}-V_{\mathrm{s} 1}$ correlation is then proposed and further validated through the results from three dynamic centrifuge model tests with silica sand no. 8 .

\section{METHODOLOGY AND APPROACH \\ Sample preparation}

The DEM simulations were performed using $\mathrm{PFC}^{3 \mathrm{D}}$ (particle flow code in three dimensions) (Itasca, 2008). In this study, around 21000 polydisperse spherical particles with diameters ranging from $0.15 \mathrm{~mm}$ to $0.20 \mathrm{~mm}$ were randomly generated in a cylindrical region (diameter $4 \mathrm{~mm} \times$ height $8 \mathrm{~mm}$ ) with rigid frictionless walls. The particle size distribution of an assembly is shown in Fig. 1. The radius expansion method was adopted to facilitate the creation of an initially isotropic sample. Each particle was prescribed with properties including a radius, density, contact stiffness and coefficient of contact friction. The Hertz-Mindlin contact model was employed in this study as it is suitable for simulating pressure-dependent behaviour at small strain (Sadd et al., 1993; Itasca, 2008; Wang \& Mok, 2008). The gravitational force was neglected in this analysis. Unless otherwise stated, the parameters used in the model are listed in Table 1.

Once the DEM assembly has been generated, a numerical servo-control mechanism specially written for the HertzMindlin contact model was implemented to compress the specimen to reach a desired isotropic stress state. The minimum void ratio $\left(e_{\min }\right)$ was obtained by setting a low initial value (e.g. $e \leqslant 0.333$ ) with the inter-particle friction set to zero at the assembly generation stage, followed by a number of numerical cycles until the isotropic stress reached $90 \%$ of the required stress. The inter-particle friction was then switched to the required contact friction value and maintained until the assembly reached an equilibrium state (the ratio of the mean unbalanced force to the mean contact force $\bar{f}^{\mathrm{u}} / \bar{f}^{\mathrm{c}} \leqslant 10^{-3}$ ) at the desired stress. The maximum void ratio $\left(e_{\max }\right)$ was obtained by assigning a large initial value (e.g. $e \geqslant 0.905)$ with an inter-particle friction of 0.50 , and by preventing particle spin when the assembly was initially generated. Particle rotation was then permitted when

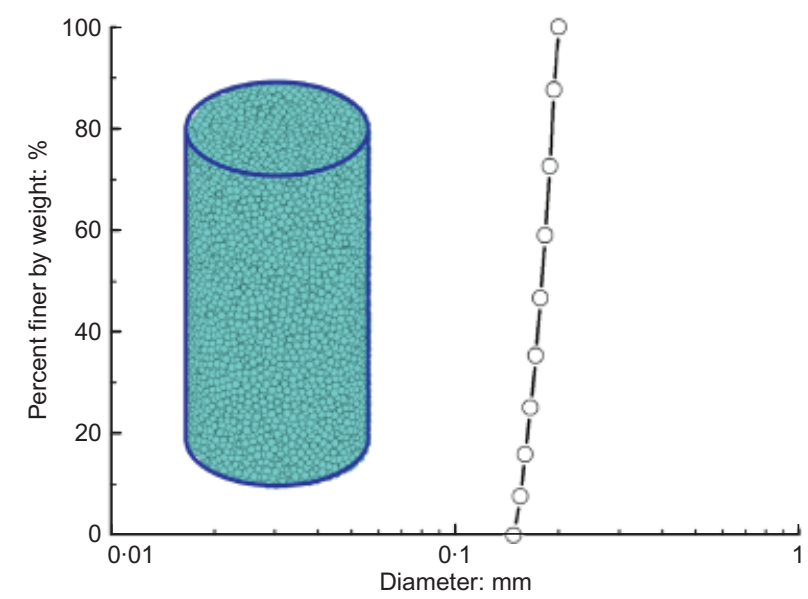

Fig. 1. Particle size distribution selection for DEM simulations; inset diagram is the initially generated sample of spheres

Table 1. Model parameters used in DEM simulations

\begin{tabular}{l|c}
\hline Parameters & Value \\
\hline Particle shear modulus, $G_{\mathrm{g}}: \mathrm{GPa}$ & $1 \cdot 0$ \\
Particle friction coefficient, $\mu_{\mathrm{g}}$ & $0 \cdot 50$ \\
Particle Poisson ratio, $v_{\mathrm{g}}$ & $0 \cdot 20$ \\
Particle density, $\rho_{\mathrm{g}}: \mathrm{kg} / \mathrm{m}^{3}$ & 2630 \\
Particle diameter, $d: \mathrm{mm}$ & $0 \cdot 15 \sim 0 \cdot 20$ \\
Model height, $H: \mathrm{mm}$ & 8 \\
Model diameter, $D: \mathrm{mm}$ & 4 \\
\hline
\end{tabular}


the isotropic stress level reached $90 \%$ of the required stress. For the other relatively loose samples, the inter-particle friction coefficients were adjusted by trial and error during the assembly generation stage to facilitate the sample reaching the desired void ratio. Similar procedures for sample preparation can be found in Salot et al. (2009), Thornton \& Zhang (2010) and Gong et al. (2012). In this study, the $e_{\max }$ and $e_{\min }$ at $100 \mathrm{kPa}$ confining pressure are 0.809 and 0.585 , respectively. These values are very close to that of the traditional random loose packing (RLP) and random close packing (RCP) for monodisperse spheres, where the $e_{\max }$ and $e_{\min }$ are 0.818 and 0.577 (Song et al., 2008; Silbert, 2010). The relative density $\left(D_{\mathrm{r}}\right)$ of the DEM specimens is expressed as

$$
D_{\mathrm{r}}=\frac{e_{\max }-e}{e_{\max }-e_{\min }}
$$

where $e$ is the void ratio after isotropic consolidation.

\section{Undrained stress-controlled cyclic triaxial test}

After isotropic consolidation, a series of undrained cyclic triaxial tests with various CSRs were simulated in a stresscontrolled manner for each numerical specimen, by applying a number of sinusoidal cyclic stress loadings until initial liquefaction occurred. The velocities of the boundaries were adjusted in such a way that the cyclic deviator stress followed a sinusoidal cyclic stress history while the specimen volume remained constant. The input frequency of the cyclic loading was chosen as $1000 \mathrm{~Hz}$ in this study. We performed a comparison among the cyclic-induced boundary responses and backbone curves of another numerical sample (with fewer particles) at various frequencies $(1 \mathrm{~Hz} \sim 5000 \mathrm{~Hz})$. The results indicated that the cyclic behaviours of the DEM specimen are essentially not sensitive to the input frequency when its value is less than $2000 \mathrm{~Hz}$. This threshold is higher than the experimental findings from a cylindrical sand sample $38 \mathrm{~mm}$ in diameter and $78 \mathrm{~mm}$ high (Bolton \& Wilson, 1989) owing to the much smaller size of the DEM specimen in the present study. These simulations were performed using four workstations (each with $3 \cdot 2 \mathrm{GHz}$ Intel $\mathrm{CPU}$ and $8-$ 32 GB memory) and one computer cluster (with eight nodes, each with $4 \times 2 \cdot 26 \mathrm{GHz}$ Intel CPU and $16 \mathrm{~GB}$ memory) over a few years.

The equivalent excess pore-water pressure $(u)$ is evaluated under an assumption of fully saturated conditions, by taking the difference between the initial effective confining pressure $\left(\sigma_{\mathrm{r} 0}^{\prime}\right)$ at the beginning of shearing and the current effective stress $\left(\sigma_{\mathrm{r}}^{\prime}\right)$, that is, $u=\sigma_{\mathrm{r} 0}^{\prime}-\sigma_{\mathrm{r}}^{\prime}$ (e.g. $\mathrm{Ng} \&$ Dobry, 1994;

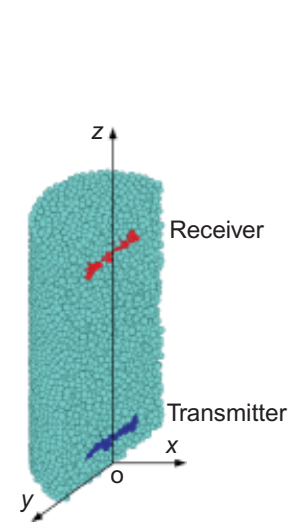

(a)

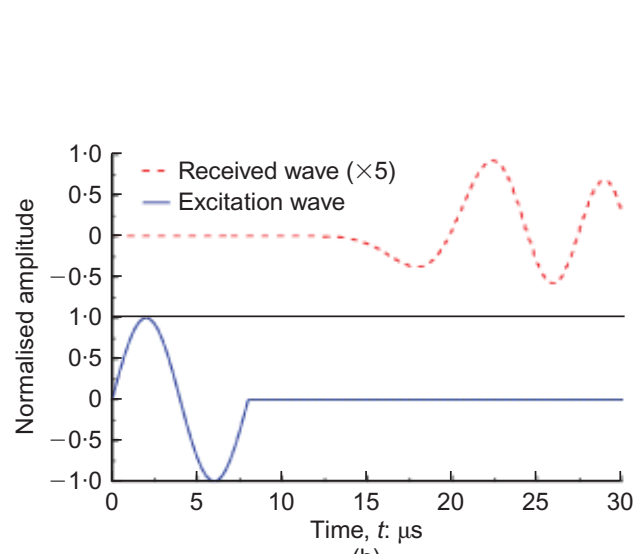

(b)

b)
Sitharam et al., 2009; Yimsiri \& Soga, 2010). The excess pore-water pressure ratio is then defined as $U=u / \sigma_{\mathrm{m} 0}^{\prime}$, where $\sigma_{\mathrm{m} 0}^{\prime}$ is the initial mean confining pressure. Initial liquefaction is said to have occurred when the effective stress becomes zero $(U=1 \cdot 0)$ owing to the build-up of excess pore-water pressure.

\section{Shear wave velocity measurement}

The measurement of shear wave velocity was implemented using a pair of virtual 'disc elements' fully embedded in the sample, as shown in Fig. 2(a). In the DEM model, a group of particles (a circular disc with around 100 particles) in a certain region were selected as a transmitter and another one as a receiver. The excitation wave was created by applying a velocity pulse to the transmitter in the $x$ direction, which would immediately be transferred to neighbouring particles and subsequently propagated through the whole sample. The wave propagation could be visualised by way of the velocity field. Once the disturbance reached the receiver, the average velocity in the same direction would be picked up. Fig. 2(b) plots the normalised velocity-time histories of both the excitation and received waves. The latter is scaled up by five times for better visualisation. The Cartesian coordinate system $o x y z$, as shown in Fig. 2(a), is used in this paper for all cases analysed in 3D space. The longitudinal direction of the numerical sample is chosen as the $z$-axis, and the $x$-axis and the $y$-axis directions are the radial directions.

Note that before performing the shear wave propagation, it is essential to ensure that the sample has reached a further equilibrium state $\left(\bar{f}^{\mathrm{u}} / \bar{f}^{\mathrm{c}} \leqslant 10^{-9}\right)$. To do this, the coefficient of local damping was set to a relatively high level (e.g. 0.9) with the purpose of dissipating energy more efficiently and saving computational time. After more numerical cycles, the velocities of the particles would eventually become low enough compared to the excitation magnitude. The damping factor was then reduced to zero at the end of this stage for the shear wave analysis.

The $V_{\mathrm{s}}$ is calculated using the wave travel time $(t)$ and the distance of the travel path $\left(L_{\mathrm{TR}}\right)$, in exactly the same way as it is in a laboratory test with bender elements (Clayton, 2011).

$$
V_{\mathrm{s}}=\frac{L_{\mathrm{TR}}}{t}
$$

The travel distance of the shear wave component is generally taken as the tip to tip distance between the transmitter and receiver. However, the determination of the

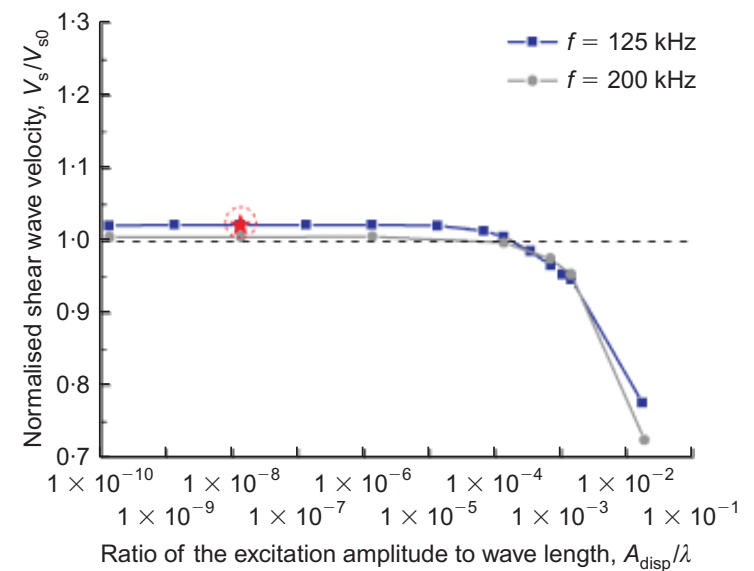

(c)

Fig. 2. Measurement of shear wave velocity: (a) half of the DEM model for wave propagation; (b) transmitted wave and received wave; (c) choice of velocity amplitude for shear wave excitation 
travel time is much less straightforward (Jovicic et al., 1996; Viana da Fonseca et al., 2009). A number of methods have been commonly used, ranging from the simplest approach based on the immediate observation of the wave traces (e.g. first arrival and peak to peak), to more elaborate signal processing techniques (e.g. cross-correlation analysis, wavelet analysis and phase detection analysis). In this study, the cross-correlation analysis was adopted, owing to its superiority in both determining the travel time and identifying the similarities between two signals (Santamarina \& Fratta, 1998).

The velocity amplitude used in this paper for the shear wave propagation was selected by comparing the measured $V_{\mathrm{s}}$ from the above-mentioned wave propagation, to the actual value $V_{\mathrm{s} 0}$ interpreted from a boundary measurement of the small strain shear modulus $G_{0}\left(V_{\mathrm{s} 0}=\sqrt{G_{0} / \rho}\right)$. Fig. 2(c) plots the variation of the normalised shear wave velocity $V_{\mathrm{s}} / V_{\mathrm{s} 0}$ against the ratio of the excitation amplitude to the wave length $A_{\text {disp }} / \lambda$. When the excitation amplitude is too high, $V_{\mathrm{s}}$ is smaller than $V_{\mathrm{s} 0}$. When $A_{\text {disp }} / \lambda$ reduces to $10^{-5}$, then shear wave velocity becomes very close to the boundary measurement $\left(V_{\mathrm{s}} / V_{\mathrm{s} 0}=1\right)$. In the figure, the value indicated by the single star point $\left(A_{\text {disp }} / \lambda=10^{-8}\right)$ is used in this paper. To ensure a linear elastic wave propagation without inducing any frictional work, the authors suggest the ratio of the displacement amplitude of the excitation wave to the mean particle diameter $A_{\text {disp }} / d_{50}<10^{-4}$ (Xu et al., 2012) or $A_{\text {disp }} / \lambda<10^{-5}$, according to Fig. 2(c).

\section{RESULTS AND OBSERVATIONS}

Over 108 numerical samples were cyclically sheared from different initial states (i.e. in terms of relative density, initial confining pressure, particle shear modulus, particle Poisson ratio and inter-particle friction) to initial liquefaction under various CSRs, where the excess pore-water pressure ratio $U$ reaches $1 \cdot 0$. The onset of liquefaction is illustrated through both macroscopic and microscopic criteria. The key microparameters that govern the magnitude of CRR are identified, and a micro-scale CRR- $V_{\mathrm{s} 1}$ correlation is then proposed and further verified.

\section{Cyclic-induced liquefaction: from macro to micro}

The macroscopic liquefaction phenomena are observed for all numerical simulations in this study. A typical example is presented in Fig. 3, which shows the variation of axial strain and excess pore-water pressure ratio $U$ with the number of cycles $(N)$ for an isotropically consolidated sample at a confining pressure of $100 \mathrm{kPa}\left(D_{\mathrm{r}}=50 \cdot 4 \%, \mathrm{CSR}=0 \cdot 125\right)$. The inset of this figure illustrates the stress-controlled deviator stress during the cyclic loading. As observed from the figure, with the application of constant cyclic stress, both the cyclic axial strain magnitude and excess pore-water pressure ratio only increase slightly after the first 50 cycles; the axial strain magnitude then increases dramatically when the numerical specimen approaches initial liquefaction after 64 cycles, owing to the development of excess pore-water pressure. In a qualitative sense, this simulation captures the realistic behaviour of sand liquefaction phenomenon observed in laboratory experiments (Seed \& Lee, 1966; Zhou \& Chen, 2005).

To explore the underlying micro-mechanism of liquefaction, the mechanical coordination number $\left(C_{\mathrm{n}}\right)$ proposed by Thornton (2000) is adopted herein. It is calculated as an average coordination number, but excludes particles with only one or zero contacts that are not contributing to the stable state of stress. It is expressed as

$$
C_{\mathrm{n}}=\frac{2 N_{\mathrm{c}}-N_{\mathrm{b} 1}}{N_{\mathrm{b}}-N_{\mathrm{b} 0}-N_{\mathrm{b} 1}}
$$

where $N_{\mathrm{b}}$ and $N_{\mathrm{c}}$ are the total number of particles and contacts respectively; $N_{\mathrm{b} 1}$ and $N_{\mathrm{b} 0}$ are the number of particles with only one or no contacts, respectively. Fig. 4 plots the evolution of $C_{\mathrm{n}}$ with the number of cycles towards initial liquefaction. Note that the data are taken at the end of each cycle (see the inset of Fig. 3), except that more data points are taken in the last incomplete cycle when the specimen was very close to initial liquefaction. After the first cycle, $C_{\mathrm{n}}$ decreases from 4.83 to 4.79 , and then remains almost constant until the 50th cycle. After that, the number decreases sharply to about $4 \cdot 0$, which is the minimum requirement for a stable three-dimensional deposit of frictional spherical particles (Edwards, 1998).

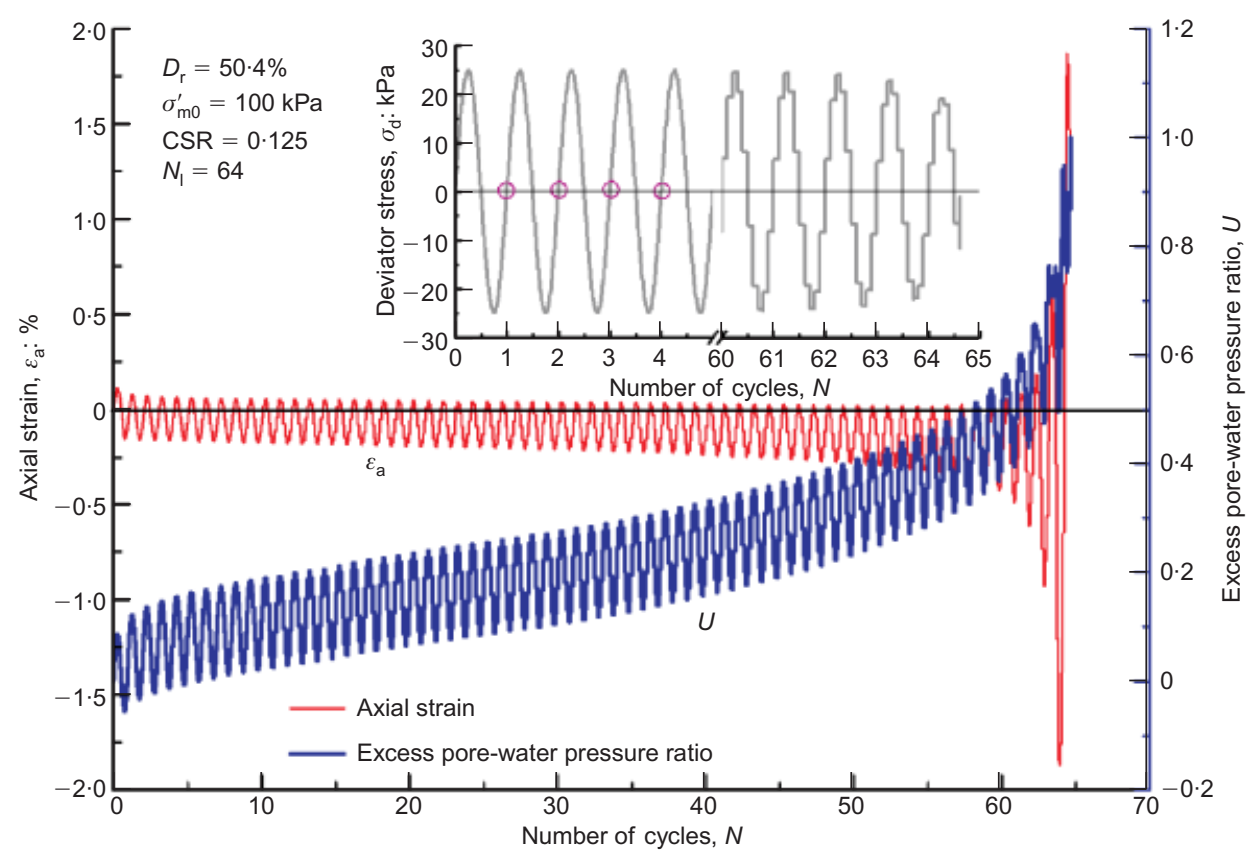

Fig. 3. Variation of axial strain and excess pore-water pressure ratio with number of cycles for a confining pressure of $100 \mathrm{kPa}\left(D_{\mathrm{r}}=\mathbf{5 0} \cdot 4 \%, \mathrm{CSR}=\mathbf{0} \cdot 125\right)$; inset diagram shows the stresscontrolled cyclic loading 


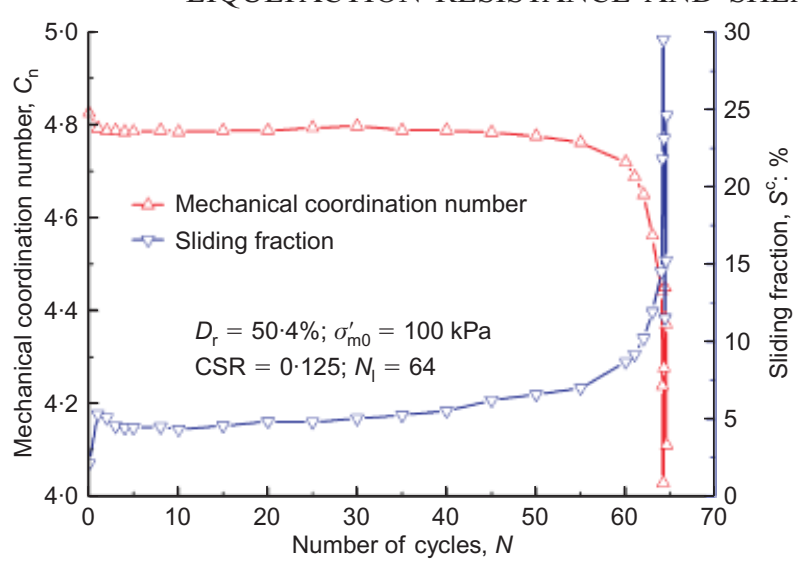

Fig. 4. Evolution of mechanical coordination number and sliding fraction with the number of cycles for a confining pressure of $100 \mathrm{kPa}\left(D_{\mathrm{r}}=50 \cdot 4 \%, \mathrm{CSR}=0 \cdot 125\right)$

During the liquefaction process, the number of interparticle contacts changes as a result of contact destruction and contact reorientation. The former can be characterised by the contact sliding fraction $\left(S^{\mathrm{c}}\right)$, which is defined as the ratio of sliding contact number to the total number of contacts. Fig. 4 also shows the variation of $S^{c}$ with the number of cycles. At the beginning of the shearing, the magnitude of $S^{\mathrm{c}}$ is only $2.09 \%$, attributed to the isotropic consolidation, and it increases to $5.31 \%$ after the first cycle. It then increases rapidly from $6 \cdot 57 \%$ at the 50 th cycle up to $29 \cdot 5 \%$ at the end of shearing. At this stage, the majority of the stored energy in the sample is dissipated. Moreover, the redundancy index $\left(I_{\mathrm{R}}\right)$ can also be used to illustrate the occurrence of liquefaction. It is defined as the ratio of the number of constraints to the number of degrees of freedom in the system, and expressed as $I_{\mathrm{R}}=C_{\mathrm{n}}\left(3-2 S^{\mathrm{c}}\right) / 12$ by Gong et al. (2012). If $I_{\mathrm{R}}>1$, this indicates a redundant system, otherwise there is a non-redundant one. In this study, for the sample shown in Fig. 4, a redundant system is identified at the initial state $\left(I_{\mathrm{R}}=1 \cdot 190\right)$, and an unstable state is recognised when initial liquefaction occurs $\left(I_{\mathrm{R}}=0 \cdot 809\right)$.

To further assess the evolution of contact reorientation during the cyclic loading, three deviator anisotropy coefficients are used in contact normals $\left(a_{\mathrm{d}}^{\mathrm{r}}\right)$, normal contact forces $\left(a_{\mathrm{d}}^{\mathrm{n}}\right)$ and tangential contact forces $\left(a_{\mathrm{d}}^{\mathrm{t}}\right)$, which are based on a second-order fabric tensor, a second-order normal contact force tensor and a second-order tangential contact force tensor, respectively (Chantawarangul, 1993; Sitharam et al., 2009). For an isotropic assembly, all these three coefficients are zero. The system is anisotropic otherwise. Fig. 5 presents their evolution with the number of cycles. It

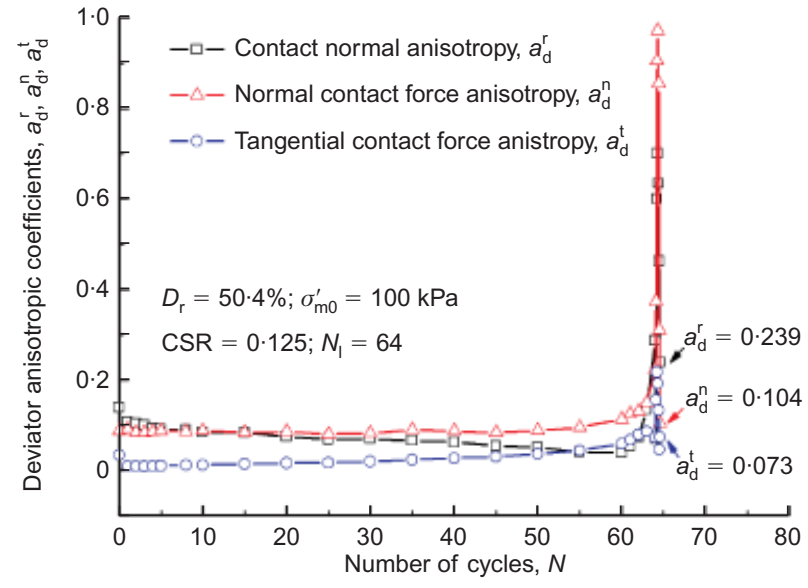

Fig. 5. Variation of deviator anisotropic coefficients with number of cycles for a confining pressure of $100 \mathrm{kPa}\left(D_{\mathrm{r}}=50 \cdot 4 \%\right.$, $\mathrm{CSR}=0 \cdot 125$ )

can be observed from the figure that all three coefficients $a_{\mathrm{d}}^{\mathrm{r}}, a_{\mathrm{d}}^{\mathrm{n}}$ and $a_{\mathrm{d}}^{\mathrm{t}}$ increase (from their initial values of $0 \cdot 14,0 \cdot 09$ and $0 \cdot 03$, respectively) dramatically (to $0 \cdot 239,0 \cdot 104$ and 0.073 , respectively) when initial liquefaction occurs. The final magnitude of each coefficient indicates that the geometrical anisotropy $\left(a_{\mathrm{d}}^{\mathrm{r}}=0.239\right)$ at liquefaction dominates the mechanical anisotropy $\left(a_{\mathrm{d}}^{\mathrm{n}}=0 \cdot 104 ; a_{\mathrm{d}}^{\mathrm{t}}=0.073\right)$, which is the signature of anisotropy at liquefaction (Guo \& Zhao, 2013).

Figure 6 plots the 2D visualisation (in the $x z$ plane) of the contact force network changing as initial liquefaction approaches. The thickness of the lines is proportional to the magnitude of the contact forces of each contact. As the number of cycle increases, the contact force network gradually becomes looser and weaker. The value of average normal contact force is $3.49 \times 10^{-3} \mathrm{~N}$ at the initial state, and reduces to $3.78 \times 10^{-4} \mathrm{~N}$ at the liquefaction state, where the contact force network becomes insufficient to sustain any more shearing. Fig. 7 illustrates the influence of the CSR on the evolution of the mechanical coordination number towards initial liquefaction. The number of cycles is normalised by the number of cycles when initial liquefaction occurs $\left(N_{1}\right)$. Both cases show that $C_{\mathrm{n}}$ remains fairly constant until $N / N_{1}>0 \cdot 6$.

Liquefaction resistance and shear wave velocity

Figure 8 shows the relationship between CSR and $N_{1}$ for various relative densities at a confining pressure of $100 \mathrm{kPa}$, together with $V_{\mathrm{s}}$ measurement at the initial isotropic state.

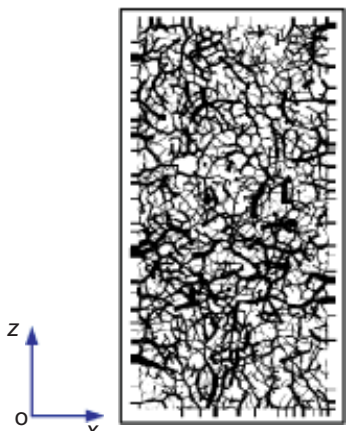

(a)

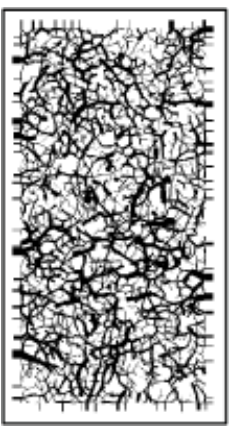

(b)

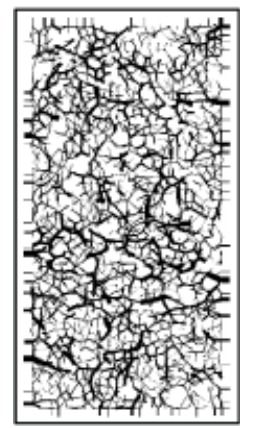

(c)

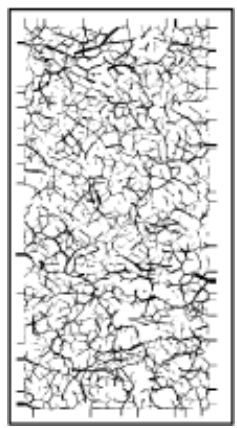

(d)

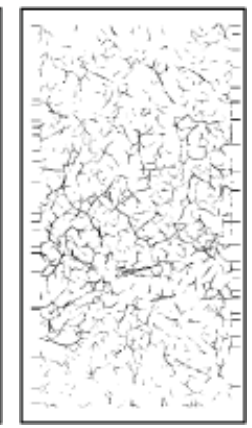

(e)

Fig. 6. Contact force network at initial state, 50th, 60th and 64th cycles and initial liquefaction $\left(f_{\max }^{\mathrm{c}}=0.02078 N\right) \quad\left(D_{\mathrm{r}}=50 \cdot 4 \%, \quad C S R=0.125\right): \quad$ (a) $N=0 ;$ (b) $N=50 ; \quad$ (c) $N=60 ;$ (d) $N=64$; (e) $N=64 \cdot 5$ 


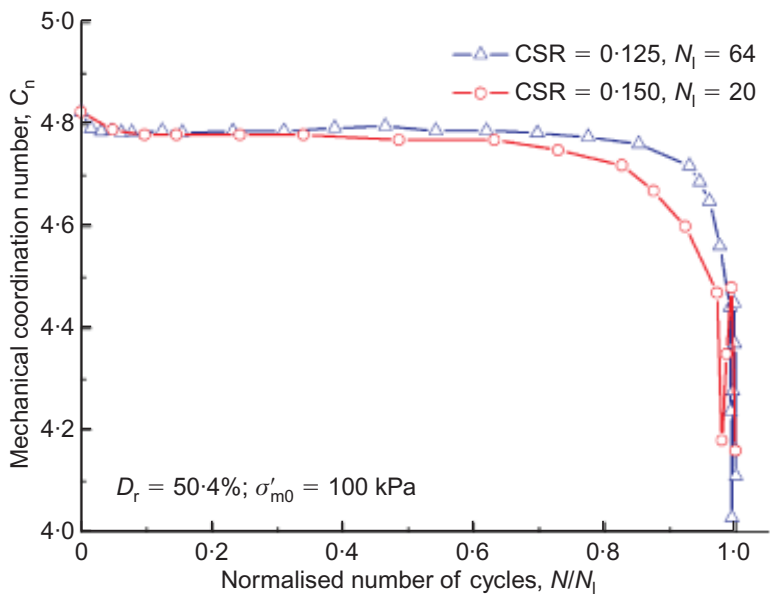

Fig. 7. Influence of cyclic stress ratio on the evolution of mechanical coordination number for a confining pressure of $100 \mathrm{kPa}$ $\left(D_{\mathrm{r}}=\mathbf{5 0} \cdot \mathbf{4} \%\right)$

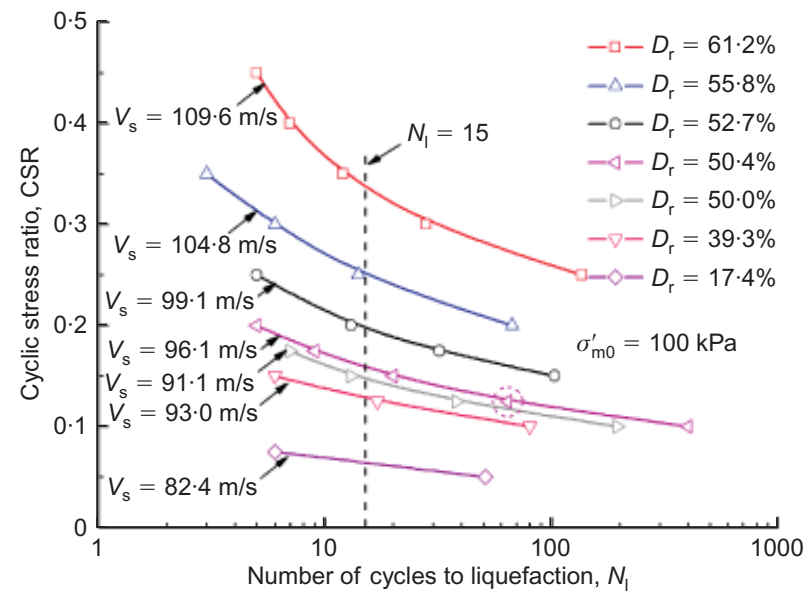

Fig. 8. Curves of CSR against number of cycles to liquefaction for a confining pressure of $100 \mathrm{kPa}$

From the figure, it is evident that the CSR curves move downwards significantly as $D_{\text {r }}$ reduces, which means that a much smaller CSR is required to liquefy a looser soil sample. This suggests that the DEM simulations have captured quantitatively the effect of $D_{\mathrm{r}}$ on liquefaction potential observed in laboratory experiments (e.g. Zhou et al., 2010). The magnitude of CRR is taken as the value of CSR when $N_{1}$ equals 15 for each specimen. As expected, CRR also decreases as $D_{\mathrm{r}}$ decreases. It is worth noting that, in Fig. 8, the single point specified by a dotted circle corresponds to the results previously presented in Figs 3-6.

To develop the liquefaction evaluation chart, the CRRs obtained from Fig. 8 are plotted against the converted stresscorrected shear wave velocity measured at the initial state of each sample in Fig. 9. The data from tests under a different confining pressure $\left(\sigma_{\mathrm{m} 0}^{\prime}=50 \mathrm{kPa}\right)$ are also added to the figure. All the data fall into a very narrow band. This demonstrates that there exists a unique $\mathrm{CRR}-V_{\mathrm{s} 1}$ correlation for a granular soil at the macro scale. The data are then fitted using the following equation

$$
\mathrm{CRR}=\alpha\left(\frac{\rho_{\mathrm{g}}\left(V_{\mathrm{s} 1}\right)^{2}}{P_{\mathrm{a}}}\right)^{\beta / 2}
$$

where $\alpha=7.218 \times 10^{-8}$ and $\beta=5.339$ (with the fitting correlation coefficient $R^{2}=0.98$ ) are material constants related to the particle mechanical properties; $P_{\mathrm{a}}$ is the atmo-

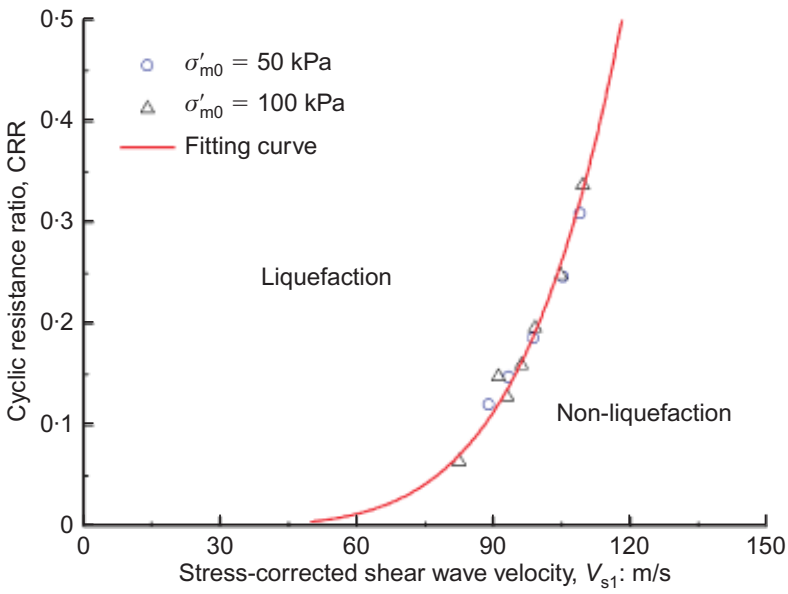

Fig. 9. Correlation between liquefaction resistance and shear wave velocity

spheric pressure (typically $100 \mathrm{kPa}$ ); and $V_{\mathrm{s} 1}$ is the overburden stress-corrected shear wave velocity, which is expressed as (Robertson et al., 1992)

$$
V_{\mathrm{s} 1}=V_{\mathrm{s}}\left(\frac{P_{\mathrm{a}}}{\sigma_{\mathrm{v}}^{\prime}}\right)^{n / 2}
$$

where $\sigma_{\mathrm{v}}^{\prime}$ is the overburden stress and $n$ is the power exponent. The value of $n$ is 0.386 for the present DEM simulations (Xu et al., 2013), which is determined by performing the shear wave velocity measurement on a number of samples at different initial states, such as void ratio and confining pressure. It is lower than that of real sand (typically $n=0 \cdot 5$ ), owing to the idealised sphere-sphere Hertzian contact characteristics in this study, rather than the non-spherical contacts in real sand particles (Santamarina \& Cascante, 1996).

\section{Particle-specific CRR- $\mathrm{V}_{\text {sI }}$ correlation}

In order to further explore the fundamental micro-mechanism of the CRR $-V_{\mathrm{s} 1}$ correlation, the influence of the particle mechanical properties on both liquefaction resistance and shear wave velocity were examined. The benchmark specimen was selected as the one isotropically consolidated to a confining pressure of $100 \mathrm{kPa}$, with relative density of $50 \cdot 4 \%$. Particle properties listed in Table 1, such as particle shear modulus, particle Poisson ratio or friction coefficient, were replaced by various values at the end of the isotropic consolidation stage, and then numerically cycled until the new specimen reach its equilibrium state. During this process, no significant changes in the void ratio, mechanical coordination number and contact normal anisotropy were found. After that, these samples were brought to initial liquefaction as before.

Figure 10 shows the effects of particle Poisson ratio $v_{\mathrm{g}}$, shear modulus $G_{\mathrm{g}}$ and friction coefficient $\mu_{\mathrm{g}}$ on CSR- $N_{\mathrm{l}}$ curves. It is evident from Fig. 10(a) and Fig. 10(b) that both $G_{\mathrm{g}}$ and $v_{\mathrm{g}}$ have negligible influence on CRR, as all these curves almost overlap. For instance, the values of CRR are $0 \cdot 159,0 \cdot 167$ and $0 \cdot 162$ when the magnitudes of $G_{\mathrm{g}}$ are set as $1 \mathrm{GPa}, 5 \mathrm{GPa}$ and $10 \mathrm{GPa}$, respectively. However, a significant effect of $\mu_{\mathrm{g}}$ on CRR is found in Fig. 10(c). With the increase of particle friction coefficient from $0 \cdot 3$ to $0 \cdot 9$, the magnitude of CRR increases 3.65 times, from 0.074 to $0 \cdot 270$. The above observations imply that the inter-particle friction is the governing micro-parameter for liquefaction resistance. This is understandable as soil liquefaction is a large deformation process with energy dissipation (e.g. 


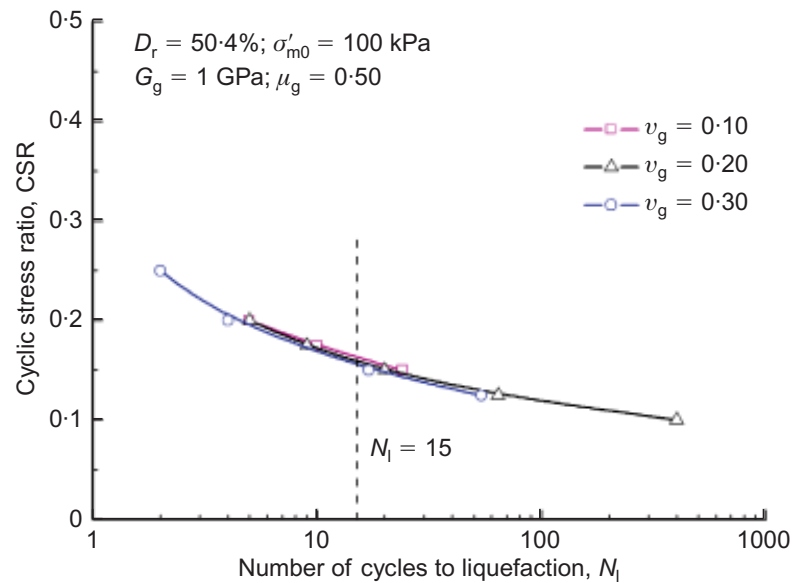

(a)

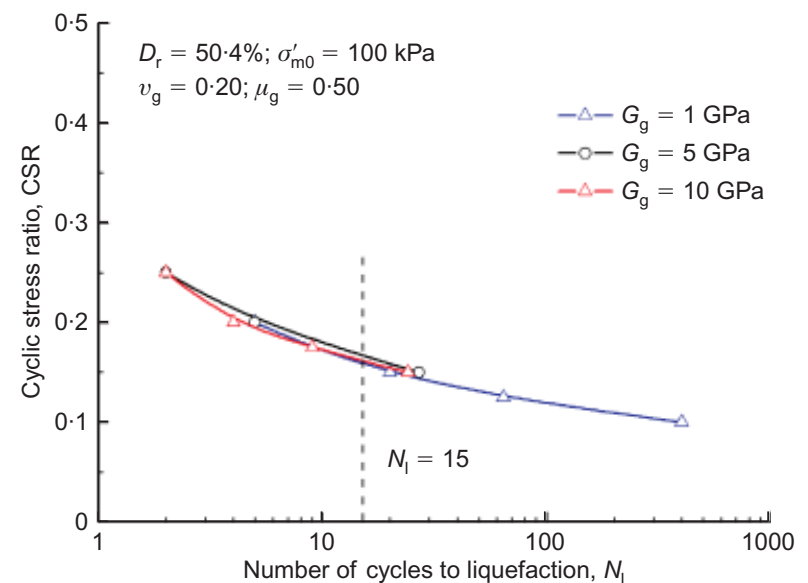

(b)

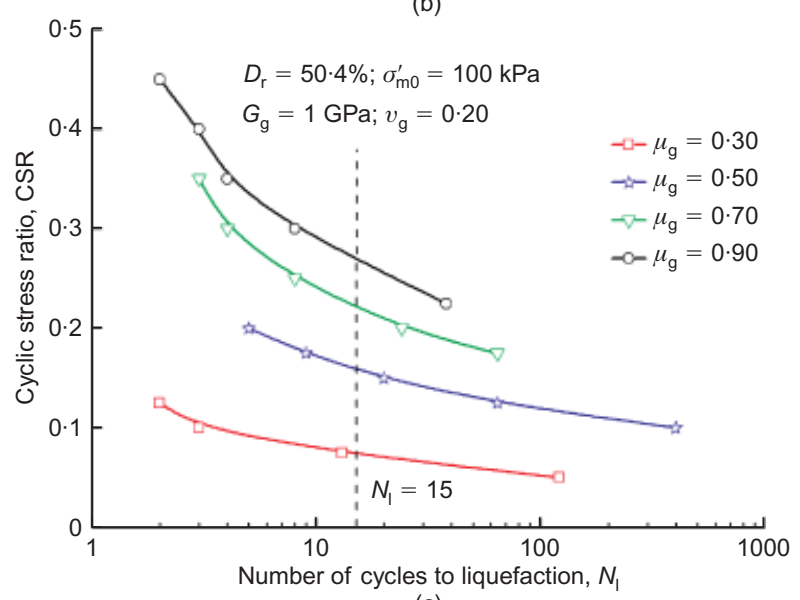

(c)

Fig. 10. Influence of particle mechanical properties on liquefaction resistance: (a) particle Poisson ratio; (b) particle shear modulus; (c) inter-particle friction

through contact sliding, as shown in Fig. 4), which is highly related to the contact frictional property. The higher the value of $\mu_{\mathrm{g}}$, the more difficult it is to induce contact sliding (Thornton, 2000), resulting in a higher CRR. These findings suggest that CRR is highly dependent on the inter-particle mobility (i.e. void ratio, coordination number, confining pressure and inter-particle friction), rather than the deformability of the soil particle itself.

Figure 11 presents the correlation between the mobilised angle of internal friction $\phi$ (both at peak $\phi_{\max }$ and critical state $\left.\phi_{\mathrm{cv}}\right)$ and inter-particle friction angle $\phi_{\mathrm{g}}$ obtained from a series of drained DEM simulations at a confining pressure of $100 \mathrm{kPa}$, together with some other results from the literature (Thornton, 2000; Yimsiri \& Soga, 2010). It is

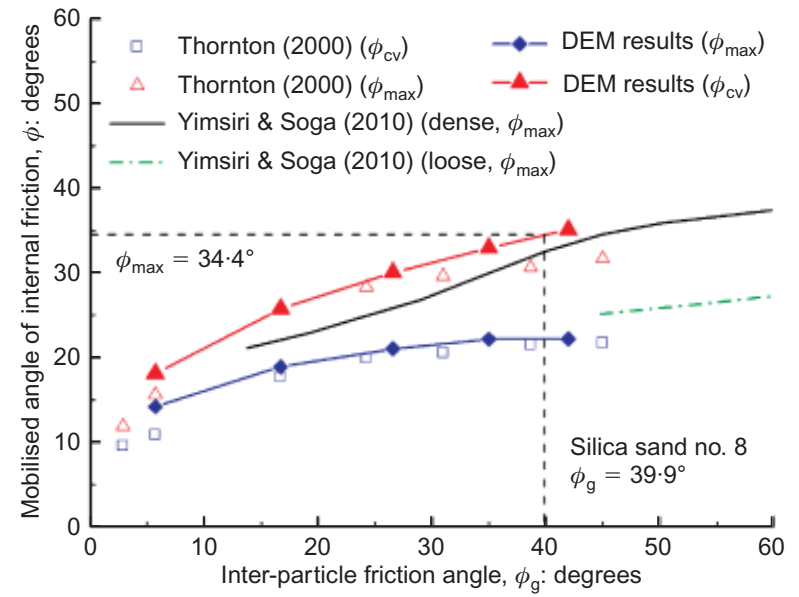

Fig. 11. Relationship between mobilised angle of internal friction and inter-particle friction angle

clearly shown that both $\phi_{\max }$ and $\phi_{\mathrm{cv}}$ from the current DEM simulations are in good agreement with those of the previous research, and the $\phi_{\max }-\phi_{\mathrm{g}}$ relationship for the present DEM assemblies with spherical particles can be expressed as

$$
\phi_{\max }=a\left(\phi_{\mathrm{g}}\right)^{b}
$$

where $a=10 \cdot 121$ and $b=0 \cdot 332$ (for $\mu_{\mathrm{g}}$ ranging from $0 \cdot 1$ to $0 \cdot 9$ ).

For the shear wave velocity of granular soils in the initial state, although not presented here, the current DEM simulations with particle shear modulus ranging from $1.0 \mathrm{GPa}$ to $50 \mathrm{GPa}$ indicate that the relationship between $V_{\mathrm{s}}$ and $G_{\mathrm{g}}$ follows a power law, with a power index of $m=0 \cdot 320$ (Xu, 2012). The influence of particle Poisson ratio $v_{\mathrm{g}}$ on $V_{\mathrm{s}}$ is found to be negligible (e.g. $V_{\mathrm{s}}$ only increases from $92 \cdot 17 \mathrm{~m} / \mathrm{s}$ to $94.68 \mathrm{~m} / \mathrm{s}$ when $v_{\mathrm{g}}$ varies from 0.1 to 0.4 with $D_{\mathrm{r}}=50 \cdot 4 \%$ and $\left.\sigma_{\mathrm{m} 0}^{\prime}=100 \mathrm{kPa}\right)$. No effect of inter-particle friction $\mu_{\mathrm{g}}$ on $V_{\mathrm{s}}$ was discovered, as the $V_{\mathrm{s}}$ measurement of the present study is within the elastic range. All these observations are consistent with the previous theoretical research based on various hypotheses (Chang et al., 1991; Santamarina \& Cascante, 1996; Yimsiri \& Soga, 2000).

It can be concluded that two independent micro-parameters, the inter-particle friction and the particle shear modulus, govern the liquefaction resistance and the shear wave velocity of granular soils, respectively. This implies that the previous CRR $-V_{\mathrm{s} 1}$ correlation shown in Fig. 9 should also be dependent on particle properties, rather than being a unique relationship for all types of granular soils.

Hence, the present authors carried out some extra DEM simulations to demonstrate the existence of particle-specific CRR $-V_{\mathrm{s} 1}$ correlations, by varying the particle shear modulus and friction coefficient. Fig. 12 presents the influence of particle shear modulus on the CRR $-V_{\mathrm{s} 1}$ correlation. The curves are the predictions from equation (5), except that the values of $\alpha$ are reduced based on the $V_{\mathrm{s}}-G_{\mathrm{g}}$ power law. Interestingly, this indicates that they are in good agreement with the results from DEM simulations at various $G_{\mathrm{g}}$ levels. For the effect of inter-particle friction, the obtained DEM data are plotted in Fig. 13, and best fitted using equation (5) by keeping $\alpha$ constant. The fitting correlation coefficients for both cases $\left(\mu_{\mathrm{g}}=0.3\right.$ and $\left.\mu_{\mathrm{g}}=0.7\right)$ are higher than 0.90 , with $\beta$ equal to $5 \cdot 135$ and $5 \cdot 440$, respectively. Therefore, from the present DEM simulations, the CRR- $V_{\mathrm{s} 1}$ correlation can be reasonably characterised by equation (5), where the coefficient $\alpha$ is dependent on $G_{\mathrm{g}}$ for a given $\mu_{\mathrm{g}}$, and the 


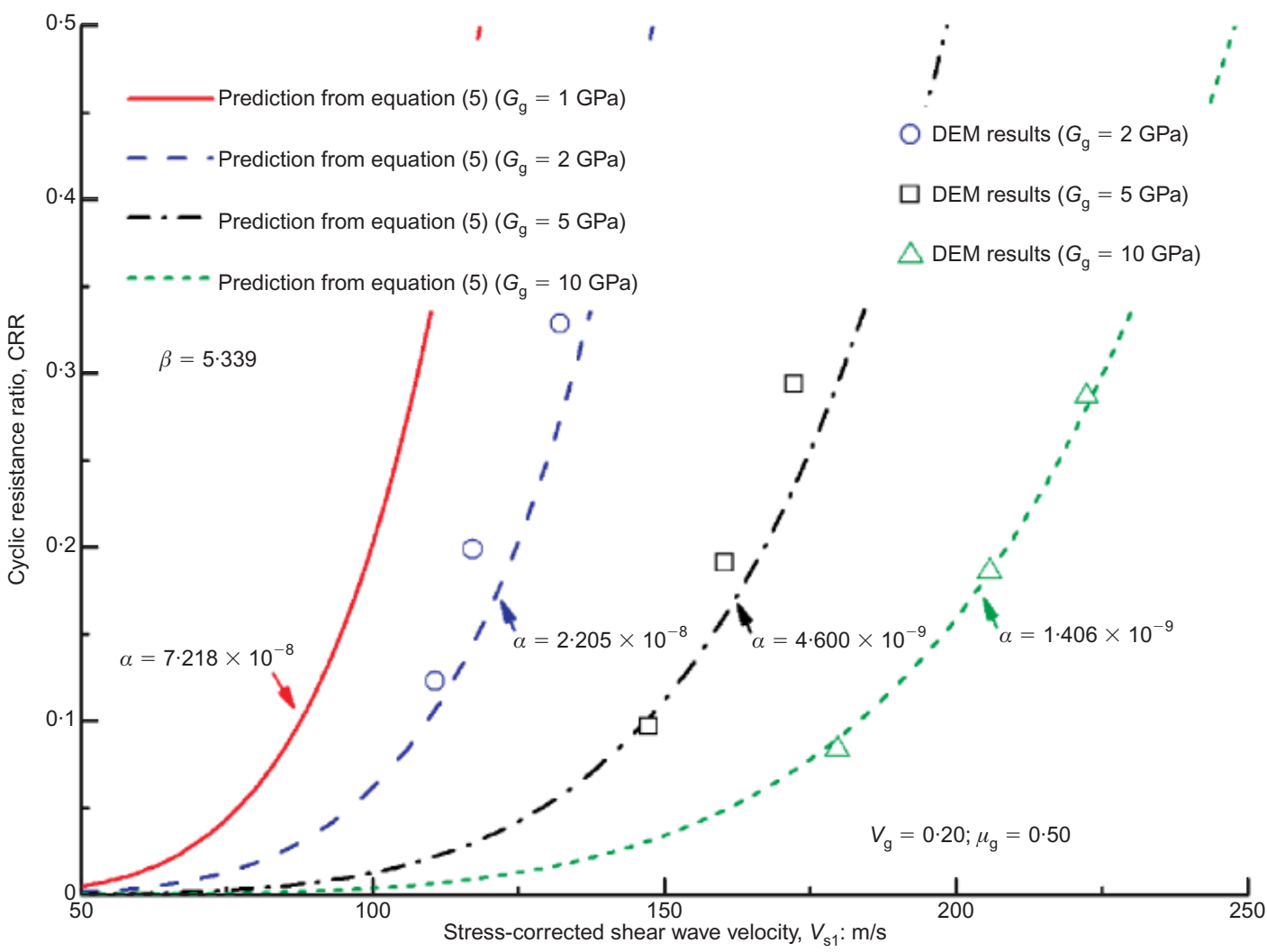

Fig. 12. Influence of particle shear modulus on $C R R-V_{s 1}$ correlation

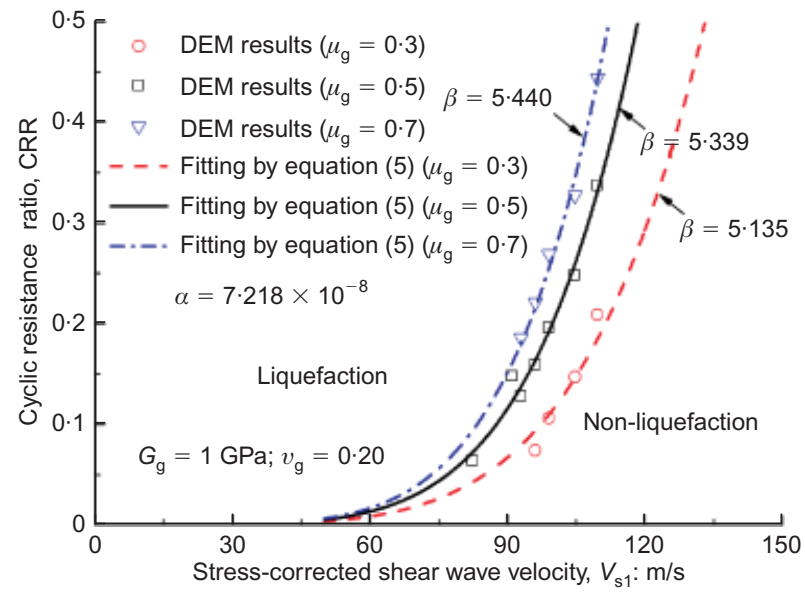

Fig. 13. Influence of inter-particle friction on CRR- $V_{s 1}$ correlation

power index $\beta$ is determined by $\mu_{\mathrm{g}}$. This can be expressed explicitly as

$$
\begin{aligned}
& \mathrm{CRR}=\frac{\alpha_{0}}{\left(G_{\mathrm{g}} / G_{\mathrm{g} 0}\right)^{m \beta}}\left[\frac{\rho_{\mathrm{g}}\left(V_{\mathrm{s} 1}\right)^{2}}{P_{\mathrm{a}}}\right]^{\beta / 2} \\
& \beta=B \arctan \left(A \mu_{\mathrm{g}}\right)+C
\end{aligned}
$$

where $\quad G_{\mathrm{g} 0}=1 \mathrm{GPa} ; \quad \alpha_{0}=7.218 \times 10^{-8} ; \quad A=4.890$; $B=0.971$; and $C=4 \cdot 191$. These two parameters may be dependent on particle shape and particle size distribution. This requires further investigation.

\section{VALIDATION AND DISCUSSION}

The present authors attempted to validate the above observation by comparing the prediction from equation (8) and the results from three dynamic centrifuge model tests (cases A, B and C) performed on silica sand no. 8 (Zhou et al., 2010). These tests were conducted in a laminar box, together with three pairs of bender elements, two pairs of earth pressure meters, eight miniature accelerometers, six miniature pore pressure transducers and three laser displacement transducers. The silica sand no. 8 is poorly graded, with a mean diameter of $0.084 \mathrm{~mm} \quad\left(e_{\max }=1.381\right.$, $\left.e_{\min }=0.721\right)$. The peak internal friction angle $\phi_{\max }$ is $34.4^{\circ}$. The relative density of the soil used in the dynamic centrifuge model varies from $61 \cdot 7 \%$ to $89 \cdot 8 \%$. From these tests, 84 liquefied data sets and 120 non-liquefied data sets were produced.

As stated previously, the use of equation (8) to characterise the $\mathrm{CRR}-V_{\mathrm{s} 1}$ correlation requires two micro-parameters: inter-particle friction and particle shear modulus. Direct measurement of these particle properties is not easy to achieve. Instead, one can calibrate them through establishing the micro-macro relationship, as shown in Fig. 11. Based on this $\phi_{\max }-\phi_{\mathrm{g}}$ relationship, equation (7), the value of $\phi_{\mathrm{g}}$ suitable for the silica sand no. $8\left(\phi_{\max }=34.4^{\circ}\right)$ is obtained as $39.9^{\circ} \quad\left(\mu_{\mathrm{g}}=0.836\right)$, leading to $\beta=5.483$ by way of equation $8(\mathrm{~b})$. For the particle shear modulus, it is calculated by comparing the $V_{\mathrm{s}}$ obtained from the lab testing using bender elements and the DEM $V_{\mathrm{s}}$ measurements from the present study at a similar relative density range, and by assuming that the real sand follows the same $V_{\mathrm{s}}-G_{\mathrm{g}}$ power law. This is reinforced by the fact that both sets of the $V_{\mathrm{s}}$ data were found to be linearly related to $D_{\mathrm{r}}$ with a similar slope. Using this method, the value of $G_{\mathrm{g}}$ was calibrated as 6.649 GPa for the silica sand no. 8 (Xu, 2012). This measured value is around four times lower than most published DEM work (Thornton, 2000; Thornton \& Zhang, 2010; Gong et al., 2012; Huang et al., 2014). This may be due to particle roughness and the imperfect shape of real sand particles (Chang et al., 1991).

Figure 14 presents the predicted $\mathrm{CRR}-V_{\mathrm{s} 1}$ curve from 


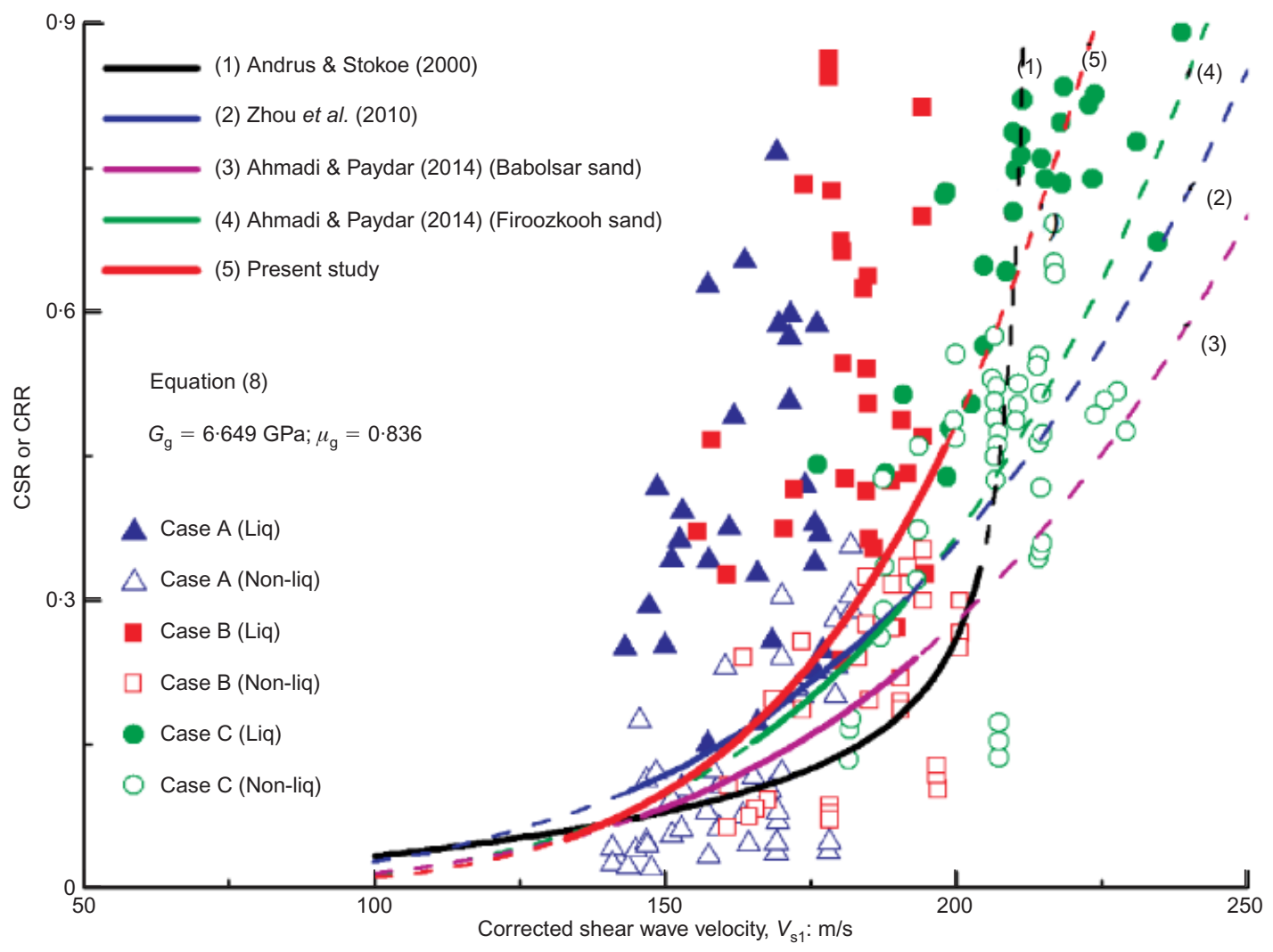

Fig. 14. Validation on the CRR- $V_{\mathrm{s} 1}$ correlation (after Zhou et al., 2010)

equation (8) using both the calibrated parameters described above and the 204 CSR data sets from the dynamic centrifuge tests (Zhou et al., 2010). The latter were converted from a series of irregular time histories recorded in centrifuge tests according to equation (1). Liquefied data are represented by solid symbols in the figure. Non-liquefied data are represented by hollow symbols. As shown in Fig. 14, the predicted curve separates almost all (about 92\%) of the liquefied data properly, and even for the non-liquefied data more than $78 \%$ of them are correctly classified. This is very similar to the previous evaluation done by Zhou et al. (2010). For the misclassification of the non-liquefied data, Zhou et al. (2010) had clarified that the initial liquefaction criterion is not always applicable to dense sand. They did observe some considerable settlement after dissipation of pore-water pressure, although these data could not be classified as liquefied according to the initial liquefaction criterion. While the correlation proposed by Andrus \& Stokoe (2000) considerably underestimates the liquefaction resistance of silica sand no. 8 within the low-velocity range, it overestimates the resistance in the high-velocity range, as shown in Fig. 14. The more recent relationship established by Ahmadi \& Paydar (2014) from two types of clean sands, namely the Firoozkooh and the Babolsar sands, are also shown in Fig. 14, and the one based on the Babolsar sand gives much less satisfactory evaluation than that of the Firoozkooh sand. These observations strongly support the fact that the proposed CRR $-V_{\mathrm{s} 1}$, which separates the liquefied and the non-liquefied data, is soil type specific.

This DEM study supports the use of equation (8) and suggests that the peak internal friction angle at the dense state can potentially be used to obtain an appropriate $\beta$ value, where the $\alpha$ value can be obtained from the shear wave velocity measurement. A satisfactory evaluation of the liquefaction potential of granular soil can then be obtained.

\section{CONCLUSIONS}

This study has explored the fundamental micromechanics of liquefaction resistance and its correlation with the shear wave velocity of granular soils, by performing a series of undrained, stress-controlled, cyclic triaxial tests on DEM specimens with various relative density, confining pressure and particle mechanical properties, together with $V_{\mathrm{s}}$ measurement at the initial state. A number of novel findings were obtained, and these are summarised as follows.

(a) The CRR- $V_{\mathrm{s} 1}$ correlation of granular soils is particle specific, and thus soil specific, and is governed by two independent micro-parameters, inter-particle friction and particle shear modulus. The proposed micro-scale CRR$V_{\text {s1 }}$ correlation, with two independent coefficients $\alpha$ and $\beta$ explicitly relating to the particle shear modulus and inter-particle friction, respectively, was validated by the outcomes from three dynamic centrifuge model test performed on silica sand no. 8 (Zhou et al., 2010). This study provides clear, yet simple, micromechanical evidence that current liquefaction evaluation with $V_{\mathrm{s}}$ alone is not sufficient, and could lead to overestimation of CRR. The effect of inter-particle friction should also be taken into account to consider the large deformation process of liquefaction in granular soils.

(b) The key micro-parameters that govern the liquefaction resistance of granular soils were identified. A systematic parametric study on DEM samples with various particle mechanical properties indicated that it is the inter-particle mobility (e.g. inter-particle friction), rather than the deformability of the soil particle itself (e.g. particle shear modulus and Poisson ratio) that determines the magnitude of CRR of granular soils. The higher the value of inter-particle friction, the more difficult it was to induce contact sliding, giving higher CRR. A micro-macro 
relationship between the inter-particle friction and the internal friction angle at peak was also developed.

(c) The micromechanics of the initial liquefaction process based on stress-controlled loading were investigated. The numerical data showed that all of the relevant micromechanical parameters, such as the mechanical coordination number, the sliding fraction, the deviator anisotropic coefficients and even the force network, remained almost constant until the number of cycles $N$ reached a relatively high portion $(>0.6)$ of the total number of cycles at which initial liquefaction occurred $N_{1}$. The onset of liquefaction is fairly sudden, leading to a dramatic change of the micro-parameters. When initial liquefaction occurs, the mechanical coordination number reduces to $4 \cdot 0$, redundancy index becomes smaller than $1 \cdot 0$, and geometrical anisotropy dominates mechanical anisotropy.

\section{ACKNOWLEDGEMENTS}

Much of the work described in this paper was supported by the National Basic Research Program of China (grant no. 2014CB047005), the National Natural Science Foundation of China (grant no. 51278451), the Zhejiang Provincial Natural Science Foundation of China (grant nos. LZ12E09001 and LY12E08011) and Erasmus Mundus External Cooperation Window (EM ECW) scholarship. These financial supports are gratefully acknowledged. The authors thank Professor Kenichi Soga and Professor Malcolm Bolton at University of Cambridge, Professor J. Carlos Santamarina at Georgia Institute of Technology and Professor M A Curt Koenders at Surrey University for their valuable exchanges of ideas and help with this study.

\section{NOTATION}

$A$ coefficient in equation (8b)

$A_{\text {disp }}$ displacement of the excitation wave

a coefficient in equation (7)

$a_{\mathrm{d}}^{\mathrm{n}}$ normal contact force anisotropy coefficient

$a_{\mathrm{d}}^{\mathrm{r}}$ contact normal anisotropy coefficient

$a_{\mathrm{d}}^{\mathrm{t}}$ tangential contact force anisotropy coefficient

$a_{\max }$ peak horizontal ground surface acceleration

$B$ coefficient in equation (8b)

$b$ power index in equation (7)

$C$ coefficient in equation (8b)

$C_{\mathrm{n}}$ mechanical coordination number

$D$ model diameter

$D_{\mathrm{r}}$ relative density

$d$ particle diameter

$d_{50}$ mean diameter of particle

$e$ void ratio after isotropic consolidation

$e_{\max }$ maximum void ratio

$e_{\min }$ minimum void ratio

$f$ excitation frequency in Fig. 2(c)

$\bar{f}^{\mathrm{c}}$ mean contact force

$f_{\max }^{\mathrm{c}}$ maximum contact force

$\bar{f}^{\mathrm{u}}$ mean unbalanced force

$G_{0} \quad$ small strain shear modulus

$G_{\mathrm{g}}$ particle shear modulus

$G_{\mathrm{g} 0}$ material constant in equation (8a)

$\boldsymbol{g}$ acceleration of gravity

$H$ model height

$I_{\mathrm{R}}$ redundancy index

$L_{\mathrm{TR}} \quad$ distance between the transmitter and receiver

$m$ power index in equation (8a)

$M_{\mathrm{w}}$ earthquake magnitude

$N$ number of cycles

$N_{\mathrm{b}}$ total number of particles

$N_{\text {b0 }}$ number of particles with no contact

$N_{\text {b1 }}$ number of particles with only one contact

$N_{c}$ total number of contacts
$N_{1}$ number of cycles to cause initial liquefaction

$n$ power exponent in equation (6)

$P_{a}$ atmospheric pressure

$r_{\mathrm{d}}$ stress reduction coefficient

$S^{\mathrm{c}}$ contact sliding fraction

$t$ wave travel time

$U$ excess pore water pressure ratio

$u$ excess pore water pressure

$V_{\mathrm{s}}$ shear wave velocity;

$V_{\mathrm{s} 0}$ shear wave velocity interpreted from a boundary measurement

$V_{\mathrm{s} 1}$ overburden stress-corrected shear wave velocity;

$\alpha$ material constant in equation (5)

$\alpha_{0}$ material constant in equation (8a)

$\beta$ material constant in equation (5)

$\varepsilon_{\mathrm{a}}$ axial strain

$\lambda$ wave length in Fig. 2(c)

$\mu_{\mathrm{g}}$ inter-particle friction coefficient

$v_{\mathrm{g}}$ particle Poisson's ratio

$\rho$ sample density

$\rho_{\mathrm{g}} \quad$ particle density

$\sigma_{\mathrm{m} 0}^{\prime}$ mean effective stress

$\sigma_{\mathrm{r}}^{\prime}$ radial effective stress

$\sigma_{\text {r0 }}^{\prime}$ radial effective stress after isotropic consolidation

$\sigma_{\mathrm{v}}^{\prime} \quad$ overburden stress

$\sigma_{\mathrm{v} 0}$ total vertical overburden stress

$\sigma_{\mathrm{v} 0}^{\prime}$ effective vertical overburden stress

$\tau_{\mathrm{av}}$ average equivalent uniform cyclic shear stress

$\phi$ internal friction angle

$\phi_{\mathrm{cv}}$ internal friction angle at critical state

$\phi_{\mathrm{g}}$ inter-particle friction angle

$\phi_{\max }$ peak internal friction angle

\section{REFERENCES}

Agnolin, I. \& Roux, J.-N. (2007). Internal states of model isotropic granular packings. III. Elastic properties. Phys. Rev. E 76, No. 6, 061304

Ahmadi, M. M. \& Paydar, N. A. (2014). Requirements for soilspecific correlation between shear wave velocity and liquefaction resistance of sands. Soil Dynam. Earthquake Engng 57, 152163.

Andrus, R. D. \& Stokoe, K. H. I. (2000). Liquefaction resistance of soils from shear-wave velocity. J. Geotech. Geoenviron. Engng 126, No. 11, 1015-1025.

Andrus, R. D., Stokoe, K. H. I. \& Juang, C. H. (2004). Guide for shear wave-based liquefaction potential evaluation. Earthquake Spectra 20, No. 2, 285-308.

Baxter, C. D. P., Bradshaw, A. S., Green, R. A. \& Wang, J. H. (2008). Correlation between cyclic resistance and shear-wave velocity for providence silts. J. Geotech. Geoenviron. Engng 134, No. 1, 37-46.

Bolton, M. D. \& Wilson, J. M. R. (1989). An experimental and theoretical comparison between static and dynamic torsional soil tests. Géotechnique 39, No. 4, 585-599, http://dx.doi.org/ 10.1680/geot.1989.39.4.585

Chang, C. S., Misra, A. \& Sundaram, S. S. (1991). Properties of granular packings under low amplitude cyclic loading. Soil Dynam. Earthquake Engng 10, No. 4, 201-211.

Chantawarangul, K. (1993). Numerical simulations of three-dimensional granular assemblies. $\mathrm{PhD}$ thesis, University of Waterloo, Canada.

Chen, Y. M., Ke, H. \& Chen, R. P. (2005). Correlation of shear wave velocity with liquefaction resistance based on laboratory tests. Soil Dynam. Earthquake Engng 25, No. 6, 461-469.

Cheng, Y. P., Bolton, M. D. \& Nakata, Y. (2004). Crushing and plastic deformation of soils simulated using DEM. Géotechnique 54, No. 2, 131-141, http://dx.doi.org/10.1680/geot.2004.54.2.131.

Clayton, C. R. I. (2011). Stiffness at small strain: research and practice. Géotechnique 61, No. 1, 5-37, http://dx.doi.org/ 10.1680/geot.2011.61.1.5.

Cundall, P. A. \& Strack, O. D. L. (1979). A discrete numerical model for granular assemblies. Géotechnique 29, No. 1, 47-65, http://dx.doi.org/10.1680/geot.1979.29.1.47. 
de Alba, P., Baldwin, K., Janoo, V., Roe, G. \& Celikkol, B. (1984). Elastic-wave velocities and liquefaction potential. Geotech. Testing $J$. 7, No. 2, 77-87.

Dobry, R., Stokoe, K. H., Ladd, R. S. \& Youd, T. L. (1981). Liquefaction susceptibility from S-wave velocity. Proceedings of the ASCE national convention: In situ tests to evaluate liquefaction susceptibility, New York. New York, NY, USA: ASCE.

Edwards, S. (1998). The equations of stress in a granular material. Physica A: Statist. Mech. Applic. 249, No. 1-4, 226-231.

Gong, G., Thornton, C. \& Chan, A. H. C. (2012). DEM simulations of undrained triaxial behavior of granular material. J. Engng Mech. 138, No. 6, 560-566.

Guo, N. \& Zhao, J. (2013). The signature of shear-induced anisotropy in granular media. Comput. Geotechnics 47, 1-15.

Hardin, B. O. \& Richart, J. F. (1963). Elastic wave velocities in granular soils. J. Soil Mech. Found. Div., ASCE 89, No. 1, 33-65.

Huang, X., Hanley, K. J., O'Sullivan, C. \& Kwok, C. Y. (2014). Exploring the influence of interparticle friction on critical state behaviour using DEM. Int. J. Numer. Analyt. Methods Geomech. 38, No. 12, 1276-1297.

Itasca (2008). Manual of particle flow code in 3-dimension, version 4. 0. Minneapolis, MN, USA: Itasca Consulting Group, Inc.

Jovicic, V., Coop, M. R. \& Simic, M. (1996). Objective criteria for determining $\mathrm{G}$ max from bender element tests. Géotechnique 46, No. 2, 357-362, http://dx.doi.org/10.1680/geot.1996.46.2.357.

Juang, C. H., Chen, C. J. \& Jiang, T. (2001). Probabilistic framework for liquefaction potential by shear wave velocity. J. Geotech. Geoenviron. Engng 127, No. 8, 670-678.

Juang, C. H., Yang, S. H. \& Yuan, H. (2005). Model uncertainty of shear wave velocity-based method for liquefaction potential evaluation. J. Geotech. Geoenviron. Engng 131, No. 10, 12741282.

Kayen, R., Moss, R. E. S., Thompson, E. M., Seed, R. B., Cetin, K. O., Kiureghian, A. D., Tanaka, Y. \& Tokimatsu, K. (2013). Shear-wave velocity-based probabilistic and deterministic assessment of seismic soil liquefaction potential. J. Geotech. Geoenviron. Engng 139, No. 3, 407-419.

Liu, N. \& Mitchell, J. K. (2006). Influence of nonplastic fines on shear wave velocity-based assessment of liquefaction. J. Geotech. Geoenviron. Engng 132, No. 8, 1091-1097.

Ng, T.-T. \& Dobry, R. (1994). Numerical simulations of monotonic and cyclic loading of granular soil. J. Geotech. Engng 120, No. 2, 388-403.

O'Sullivan, C. (2011). Particulate discrete element modelling: A geomechanics perspective. London, UK: Taylor and Francis Group.

O’Sullivan, C., Cui, L. \& O’Neill, S. C. (2008). Discrete element analysis of the response of granular materials during cyclic loading. Soils Found. 48, No. 4, 511-530.

Robertson, P. K., Woeller, D. J. \& Finn, W. D. L. (1992). Seismic cone penetration test for evaluating liquefaction potential under cyclic loading. Can. Geotech. J. 29, No. 4, 686-695.

Roy, D., Campanella, R. G., Byrne, P. M. \& Hughes, J. M. O. (1996). Strain level and uncertainty of liquefaction related index tests. In Uncertainty in the geologic environment: from theory to practice (eds C. Shackelford, P. Nelson and M. Roth), GSP No. 58, pp. 1149-1162. Reston, VA, USA: American Society of Civil Engineers.

Sadd, M. H., Tai, Q. M. \& Shukla, A. (1993). Contact law effects on wave propagation in particulate materials using distinct element modeling. Int. J. Non-linear Mech. 28, No. 2, 251-265.

Salot, C., Gotteland, P. \& Villard, P. (2009). Influence of relative density on granular materials behavior: DEM simulations of triaxial tests. Granular Matter 11, No. 4, 221-236.

Santamarina, J. C. \& Cascante, G. (1996). Stress anisotropy and wave propagation: a micromechanical view. Can. Geotech. J. 33, No. 5, 770-782.

Santamarina, J. C. \& Fratta, D. (1998). Introduction to discrete signals and inverse problems in civil engineering. Reston, VA, USA: ASCE Press.

Seed, H. B. \& Idriss, I. M. (1971). Simplified procedure for evaluating soil liquefaction potential. J. Soil Mech. Found. Div., ASCE 97, No. SM9, 1-15.

Seed, H. B. \& Lee, K. L. (1966). Liquefaction of saturated sands during cyclic loading. J. Soil Mech. Found. Div., ASCE 92, No. 6, 105-134.
Shafipour, R. \& Soroush, A. (2008). Fluid coupled-DEM modelling of undrained behavior of granular media. Comput. Geotechnics 35, No. 5, 673-685.

Silbert, L. E. (2010). Jamming of frictional spheres and random loose packing. Soft Matter 6, No. 13, 2918.

Sitharam, T. G. (2003). Discrete element modelling of cyclic behaviour of granular materials. Geotech. Geol. Engng 21, No. 4, 297-329.

Sitharam, T. G., Vinod, J. S. \& Ravishankar, B. V. (2009). Postliquefaction undrained monotonic behaviour of sands: experiments and DEM simulations. Géotechnique 59, No. 9, 739-749, http://dx.doi.org/10.1680/geot.7.00040.

Soga, K. \& O'Sullivan, C. (2010). Modeling of geomaterials behavior. Soils Found. 50, No. 6, 861-875.

Song, C., Wang, P. \& Makse, H. A. (2008). A phase diagram for jammed matter. Nature 453, No. 7195, 629-632.

Soroush, A. \& Ferdowsi, B. (2011). Three dimensional discrete element modeling of granular media under cyclic constant volume loading: a micromechanical perspective. Powder Technol. 212, No. 1, 1-16.

Tatsuoka, F. (1999). Small strain behavior of granular materials. In Mechanics of granular materials (eds M. Oda and K. Iwashita), pp. 299-308. Rotterdam, the Netherlands: Balkema.

Thornton, C. (2000). Numerical simulations of deviatoric shear deformation of granular media. Géotechnique 50, No. 1, 43-53, http://dx.doi.org/10.1680/geot.2000.50.1.43.

Thornton, C. \& Zhang, L. (2010). On the evolution of stress and microstructure during general 3D deviatoric straining of granular media. Géotechnique 60, No. 5, 333-341, http://dx.doi.org/ 10.1680/geot.2010.60.5.333.

Tokimatsu, K. \& Uchida, A. (1990). Correlation between liquefaction resistance and shear wave velocity. Soils Found. 30, No. 2, $33-42$.

Viana da Fonseca, A., Ferreira, C. \& Fahey, M. (2009). A framework interpreting bender element tests, combining time-domain and frequency-domain methods. Geotech. Testing J. 32, No. 2, $1-17$.

Wang, J., Moran, K. \& Baxter, D. P. (2006). Correlation between cyclic resistance ratios of intact and reconstituted offshore saturated sands and silts with the same shear wave velocity. $J$. Geotech. Geoenviron. Engng 132, No. 12, 1574-1580.

Wang, Y. H. \& Mok, C. M. B. (2008). Mechanisms of small-strain shear-modulus anisotropy in soils. J. Geotech. Geoenviron. Engng 134, No. 10, 1516-1530.

$\mathrm{Xu}, \mathrm{X}$. (2012). Study on the micromechanism of sand liquefaction and its evaluation. $\mathrm{PhD}$ thesis, Zhejiang University, China (in Chinese).

Xu, X., Ling, D., Cheng, Y. \& Chen, Y. (2012). Micromechanical study on shear wave velocity of granular materials using discrete element methods. In Discrete element modelling of particulate media (ed. C.Y. Wu), Special Publication 339, pp. 264-270. Cambridge, UK: Royal Society of Chemistry.

Xu, X., Cheng, Y. \& Ling, D. (2013). The influence of void ratio on small strain shear modulus of granular materials: a micromechanical perspective. In Powders and grains, Proceedings of the 7th international conference on micromechanics of granular media, Sydney, Australia (eds A. Yu, K. Dong, R. Yang and S. Luding), pp. 201-204. Sydney, Australia: AIP.

Yimsiri, S. \& Soga, K. (2000). Micromechanics-based stress-strain behaviour of soils at small strains. Géotechnique 50, No. 5, 559-571, http://dx.doi.org/10.1680/geot.2000.50.5.559.

Yimsiri, S. \& Soga, K. (2010). DEM analysis of soil fabric effects on behaviour of sand. Géotechnique 60, No. 6, 483-495, http:// dx.doi.org/10.1680/geot.2010.60.6.483.

Youd, T. L. \& Idriss, I. M. (2001). Liquefaction resistance of soils: Summary report from the 1996 NCEER and 1998 NCEER/NSF workshops on evaluation of liquefaction resistance of soils. $J$. Geotech. Geoenviron. Engng 127, No. 4, 297-313.

Youd, T. L., Idriss, I. M., Andrus, R. D., et al. (2001). Liquefaction resistance of soils: Summary report from the 1996 NCEER and 1998 NCEER/NSF workshops on evaluation of liquefaction resistance of soils. J. Geotech. Geoenviron. Engng 127, No. 10, $817-833$.

Zhao, J. \& Guo, N. (2013). Unique critical state characteristics in granular media considering fabric anisotropy. Géotechnique 63, No. 8, 695-704, http://dx.doi.org/10.1680/geot.12.P.040. 
Zhou, Y. \& Chen, Y. (2005). Influence of seismic cyclic loading history on small strain shear modulus of saturated sands. Soil Dynam. Earthquake Engng 25, No. 5, 341-353.

Zhou, Y. \& Chen, Y. (2007). Laboratory investigation on assessing liquefaction resistance of sandy soils by shear wave velocity. $J$.
Geotech. Geoenviron. Engng 133, No. 8, 959-972.

Zhou, Y., Chen, Y. \& Shamoto, Y. (2010). Verification of the soiltype specific correlation between liquefaction resistance and shear-wave velocity of sand by dynamic centrifuge test. $J$. Geotech. Geoenviron. Engng 136, No. 1, 165-177. 